\title{
Conditions for Inducing Sense of Body Ownership to Bird Avatar in Virtual Environment
}

\author{
Akimi Oyanagi*, Ren Ohmura \\ Toyohashi University of Technology 1-1 Hibarigaoka, Tenpaku, Toyohashi, Aichi, 441-8580 Japan. \\ * Corresponding author. Tel.: 080-2069-2160; email: oyanagi@usl.cs.tut.ac.jp \\ Manuscript submitted June 24, 2017; accepted August 2, 2017. \\ doi: 10.17706/jcp.13.6.596-602
}

\begin{abstract}
Previous works have reported that a body ownership illusion gives a user a feeling that an avatar, in a virtual environment, becomes their own body. While many studies investigated the cause of the body ownership illusion on a human-like avatar, few studies have been focused for an avatar whose figure is a non-humanlike, such as an animal. Thus, we conducted some experiments to investigate the specific conditions that induce a body ownership illusion to a bird avatar, and compared the strength of sense of body ownership between a human avatar and a bird avatar. We evaluated how characteristic bird features, such as flying action, short body, and sounds of flapping, affected the sense of body ownership. The results suggested that motion synchronization between a subject's real body and an avatar's one induces a sense of body ownership just the same as the case of a human-like avatar. Furthermore, we confirm that there is no difference between a human and a bird avatar in the subject's rating of body ownership. Specifically, flying action, a characteristic particular to birds, can enhance the sense of body ownership for a bird avatar, but it is also effective for a human-like avatar. Interestingly, the short height of the body and the sound of flapping do not affect the sense of body ownership.
\end{abstract}

Key words: Sense of body ownership, immersive virtual reality, body perception.

\section{Introduction}

The feeling of "this body is my own" is referred to as the sense of body ownership. Basically, our self-consciousness is attributed to our body throughout one's life. However, Botvinick \& Cohen(1998) have reported the rubber-hand illusion(RHI) where participants perceives a rubber hand as their own body by presenting synchronous multisensory-information input such as synchronous visuo-tactile stimulation [1]. Recent studies extends the RHI to immersive virtual reality(IVR) in which the virtual full body(avatar) is located as a representation of the user's body. A body ownership illusion can be induced to an avatar by presenting avatar's perspective and motion synchronization between a subject's body and an avatar as if your real body was moving.

Many studies have reported the cause of the body ownership illusion on human-like avatars such as children, mis-matched gender, mis-matched skin, and robots. Hence, this illusion can be induced even though that virtual body clearly differs from real body [2], [3]. Interestingly, the body ownership illusion affects our attitude and behaviour. For example, previous study has shown that implicit racial bias is reduced by inducing a sense of body ownership to a dark skinned avatar [2]. Therefore, it is important to investigate the possibility that we can experience the body ownership illusion regardless of avatar type for 
creating various unique events in virtual environment.

Researches of the RHI have reported that anatomical consistency (e.g. positions of body parts, body shape and skeletal structure) is important to induce a sense of body ownership [4]. However, existing research has been mainly focused on human-like avatar (i.e. Avatar has a body and skeletal structure the same as that of a human). It suggests that we can experience a body ownership illusion to a human-like avatar without depending on the avatar's appearance. IVR cannot just present only a human-like avatar but also an non-humanlike avatar, such as an animal, as a representation of our body. A non-humanlike avatar's bodily features clearly differ from a human one. Little is known about the inducing sense of body ownership with a unlike human avatar. A more recent study, and to our knowledge the only one, has reported that their experiments gives a feeling of a sense of body ownership to a cow and a coral avatar [5]. However, few studies have focused on the conditions for the inducing of a sense of body ownership for non-humanlike avatars. If a body ownership illusion can be induced with a non-humanlike avatar, it expands the capable scope of inducing the body ownership illusion. Therefore, we aim to investigate the cause of body ownership illusion to an non-humanlike avatar. First of all, we conduct an experiment where we compare a human avatar and non-humanlike avatar(bird) in order to investigate common issues concerning the sense of body ownership between avatars. The reasons why we select bird avatar as follows: (1) A bird's body structure is the same as a human (i.e. bird has wings and legs that equal to the human's limbs). Therefore it is easy to conduct an experiment that compares between a human avatar and a bird avatar. (2) Bird has features such as short size, flying ability and the sound of flapping. That is, it is unique ability for a bird.

Previous studies have shown that motion synchronization is a sufficient condition for inducing sense of body ownership. Just because the pre-existing experimental method is effective for a human-like avatar, it doesn't necessarily mean that it would be a sufficient condition for a non-humanlike avatar because our body structure differs from a bird. Therefore, we investigate to see if motion synchronization is a sufficient condition even for a bird avatar. Additionally, we evaluate how characteristic bird features, such as flying action, short body, and sounds of flapping, impact on the sense of body ownership for a bird avatar in order to investigate the unique condition for inducing the body ownership illusion to a bird avatar.

\section{Material and Methods}

\subsection{Participants}

We conducted an experiment to investigate the specific conditions that induce a body ownership illusion to a bird avatar, and compared the strength of the sense of body ownership between human and bird avatars. 20 subjects participated in the experiments, and all subjects were male. Participants did not know the purpose of the experiment.

\subsection{Method}

Participants were immersed in a virtual environment where a virtual body was located as a representation of their body. Participants manipulated an avatar by using their body movements. A virtual mirror and a female character were located in front of the avatar in the virtual room. Participants perceived the size of the avatar's body by comparing with the female character (see Fig. 1. A). They could observe the avatar's body by looking directly toward their actual body and also via the virtual mirror. Previous study has reported that the virtual mirror is a significantly effective method for inducing a sense of body ownership [6]. Additionally, participants could observe the entire body of the bird through the virtual mirror. The experiment was designed as mixed 2x3x2 conditions. Avatar (human, bird) was between-subjects variables. Movement (asynchronous versus synchronous versus flying) and character (match, not match) were within-subjects variables. 
The avatar factor was composed of two conditions that embody participants as either a human or a bird. Each avatar condition was assigned 10 participants.

The movement factor was composed of three conditions concerning the mapping of movement between the subject's body and the avatar's body. In the asynchronous condition, the virtual body moved asynchronously with respect to the movements of the participants. In the synchronous condition, avatar's motion synchronized with participants. In the flying condition, they could fly when performing a flying action gesture. When they flapped, they could move upward. They could move toward a direction by leaning. The flying speed was the same for all condition. There is an inconsistency in terms of the skeletal structure between the actual subject's body and the bird avatar. Therefore we make mapping of each of the body parts for both (see Fig. 1 (E)).

The character factor was composed of two conditions where a virtual body resembles a real body for that avatar (see Fig. 1 (D)). In the match condition with a bird avatar, it resembles an actual bird (the avatar is the size of a common bird $(20 \mathrm{~cm})$. When participants flap, the sound of flapping was produced.). In the not-match condition, the bird avatar did not resemble an actual bird (the avatar is the size of a human $(180 \mathrm{~cm})$ and the sound of flapping did not play). Contrary to the bird avatar, in the match condition with the human avatar, it resembles a human (the avatar is the size of human $(180 \mathrm{~cm})$ and sound of flapping did not play). In the not-match condition, avatar did not resemble an actual human (the avatar was the size of bird $(20 \mathrm{~cm})$. When participants flap, the sound of common flapping was produced.).

\subsection{Measurements}

We adapted the following measurements to assess the sense of body ownership. A post-experimental questionnaire can be evaluated to subjectively measure. It was designed based on previous studies [2], [3], [7], and also considered the bird avatar. Each questionnaire item was evaluated by using 1-7 Likert scale where 1 means a not at all and 7 means very much (see Table 1). Skin temperature and SCR are often used to assess physiological reaction. Skin temperature is reduced when the subject feels a sense of body ownership [8]. Previous study has shown that when subjects feel a sense of body ownership their level of stress is measured by a surrounding fire effect on their virtual body [9].

\subsection{Apparatus}

We used VR HMD Oculus DK2(960x1080 pixels and 110deg Field of View ) to embody the virtual environment (see Fig. 1(A)). Participants have viewed the VR space via this device. Participant's motion was captured by Micro Soft Kinect V2, and the movement reflects to avatar's movements (Fig. 1(B)). The experimental program ran on a pc with $4.0 \mathrm{GHz}$, GeForce 970 Ti GPU and 16GB RAM. this program was implemented by Unity Game Engine 5.3.4. As depicted by figure, participants were immersed in a virtual environment using their equipment. We used Micro soft band2 and Maxim MAXREFDES73\# to measure physiological reaction(i.e. skin temperature and skin conductance reaction (SCR)).

\subsection{Procedure}

Upon arrival at the laboratory, the investigator instructs participants on the experimental procedure. Participants completed a demographic questionnaire(age, gender, VR experience, hour spent playing video games last week). The experimenter then attached the electrodes for skin temperature and SCR, measurements and proceeded to fit the HMD. Participants confirmed the predetermined actions that must be immediately undertaken when immersed in the virtual environment. Participants stood in front of a Kinect v2.

They were immersed as human avatars and confirmed instructed actions during training session. Trial conditions were randomly represented to participants. First of all, participants performed pre-determined actions(look at both hands, look at each hand while raising them, look down on their own body and feet, 
bend the body forward, move around, flap). Once they had finished the pre-determined actions, they could move freely. Trials were $3 \mathrm{~min}$ in duration. At the end of the experiment, an virtual explosion was suddenly represented as a threating event for measuring physiological reaction. This event was inspired by previous study [7]. Participants completed a questionnaire while wearing the HMD (see Table 1). After completing this questionnaire, the next trial started immediately.

(A)

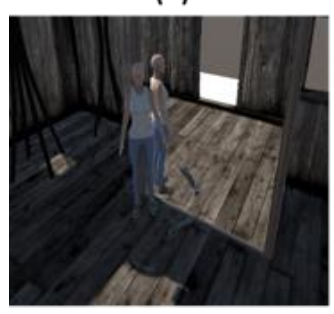

(C)

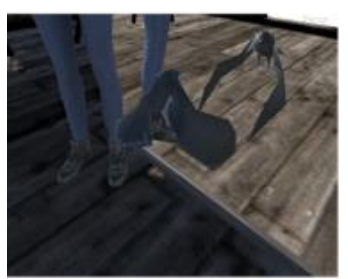

(B)

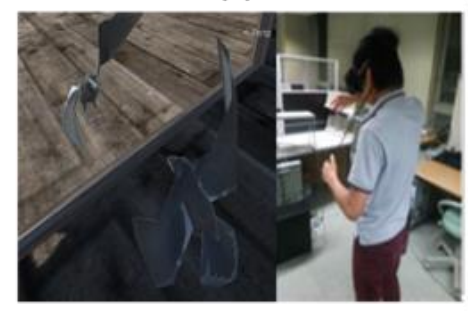

(D)

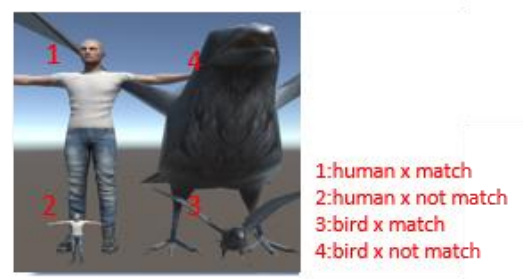

(E)

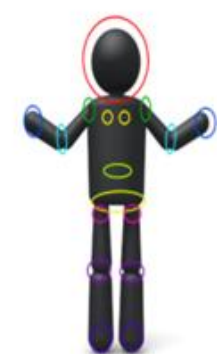

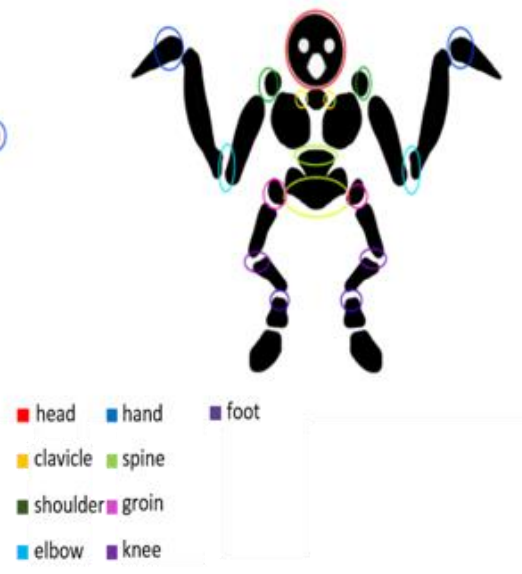

Fig. 1. Experimental overview. (A) Virtual environment (B) Real and virtual body movements synchronized. (C) During flying condition, participants could fly by performing the corresponding gesture: flap (move upward), state of both hands is parallel (stay in the sky), lean body (parallel shift toward direction in which the participants leaned). (D)Participants controlled one of mixed 2(human and bird) X2(match and not match) conditions. (E) As shown in Fig, participant's joints were corresponded with the joints of the bird avatar.

Table 1. Questionnaire in Terms of Body Ownership and Bodily Perception

\begin{tabular}{|c|c|}
\hline Variable & Question \\
\hline My Body & $\begin{array}{l}\text { How much did you feel that the virtual body you saw when you looked down at yourself was your } \\
\text { own body? }\end{array}$ \\
\hline Mirror & $\begin{array}{l}\text { How much did you feel that the virtual body you saw when you looked at yourself in the mirror } \\
\text { was your own body? }\end{array}$ \\
\hline Two Body & How much did you feel as if you had two bodies? \\
\hline Agency & How much did you feel that the virtual body followed your movement intentions? \\
\hline $\begin{array}{l}\text { Real body } \\
\text { change }\end{array}$ & $\begin{array}{l}\text { How much did you feel that your real body changed in the virtual body as if the body transforms the } \\
\text { other body? }\end{array}$ \\
\hline Features & How much did you feel that the virtual avatar resembled bird? \\
\hline Threating & How much did you feel that the real body burned when the virtual body was surrounded by fire? \\
\hline Flying & How much did you feel as if you were flying in the sky? \\
\hline Realness & Did you accept the virtual reality experience as a real experience? \\
\hline $\begin{array}{l}\text { Open } \\
\text { Questions1 }\end{array}$ & $\begin{array}{l}\text { What was your perception of the experiment (for example, I felt like I became a bird, and so on)? To } \\
\text { what degree did you feel that? }\end{array}$ \\
\hline $\begin{array}{l}\text { Open } \\
\text { Questions2 }\end{array}$ & When did the feeling of owning the virtual body appear especially strong or weak? \\
\hline $\begin{array}{l}\text { Open } \\
\text { Questions3 }\end{array}$ & Others \\
\hline
\end{tabular}



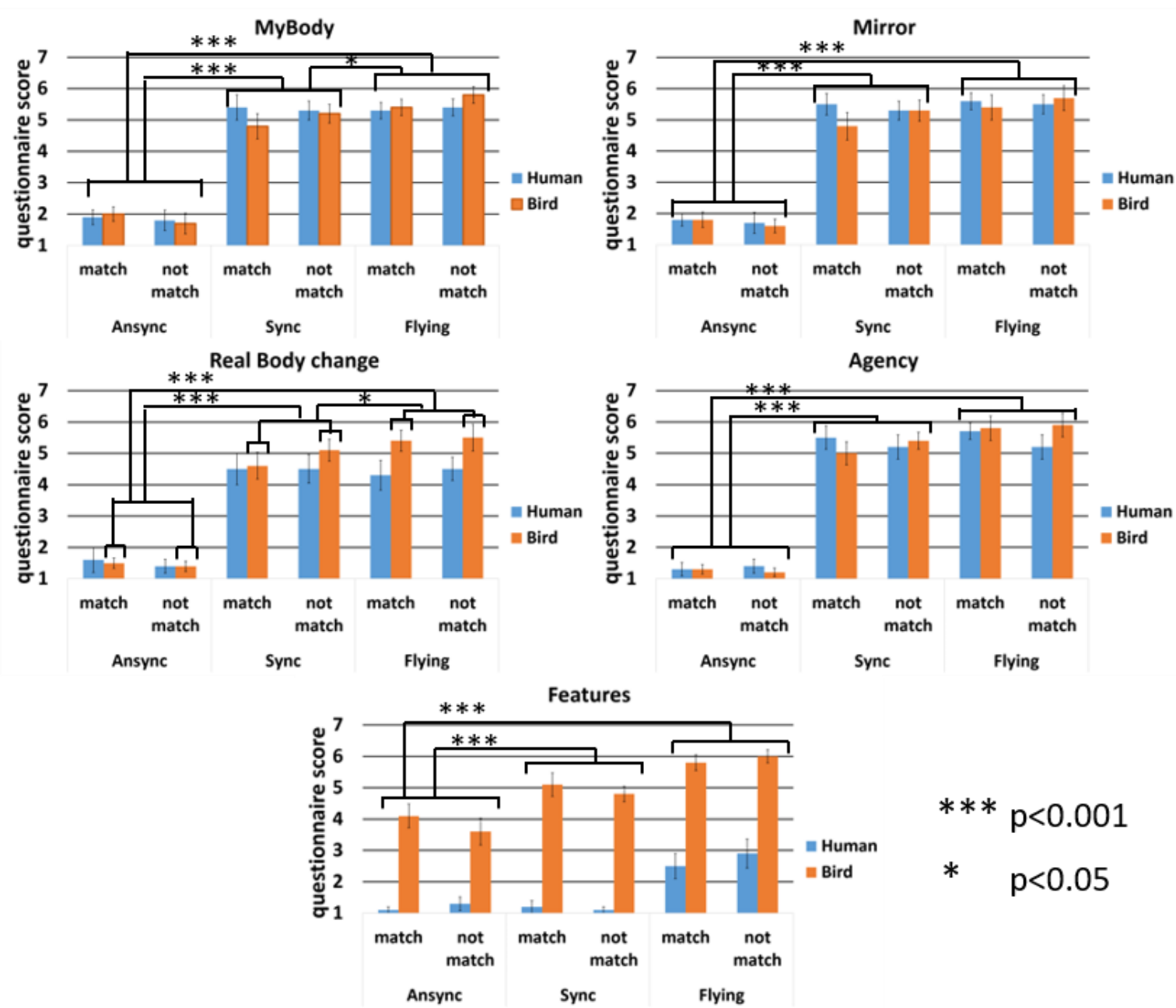

$* \quad \mathrm{p}<0.05$

Fig. 2. Results questionnaire associated with Table 1. Means are shown. Error bar shows standard error.

\section{Result}

We analyzed the results of the questionnaire by using one-way and two-way mixed ANOVA and multiple comparisons with the Holm method at the $5 \%$ significance level as a post hoc test. Physiological reaction was not used to evaluate the sense of body ownership, because we could not correctly measure the data of GSR and skin temperature.

\subsection{Body Ownership}

There was significant difference between the action factor in rating my body or mirror that directly related to the sense of body ownership (My Body $\mathrm{p}<0.001$, Mirror $\mathrm{p}<.001$ ). Synchronous condition was significantly higher than the asynchronous condition, and the flying condition was significantly higher for multiple comparison. There was no interaction.

\subsection{Body Change}

There was significant difference between movement factors $(\mathrm{p}<0.001)$. Flying condition was significantly higher than the synchronous condition and the asynchronous condition with bird avatar for multiple comparisons.

\subsection{Agency}

There was significant difference between movement factors $(\mathrm{p}<0.001)$.Flying condition and Synchronous condition two were higher than asynchronous the condition. 


\subsection{Features}

There was significant difference between movement factors $(\mathrm{p}<0.001)$. Flying condition was significantly higher than the synchronous condition and asynchronous condition for multiple comparisons.

\section{Discussion}

As a result of the analysis of the questionnaire, we confirmed that the synchronous condition was significantly higher than asynchronous condition with regard to two items(My Body, Mirror) that directly evaluate the sense of body ownership. We obtained results the same as previous works that solely focused on a humanlike avatar. Further, there was no significant difference between avatars. It suggests evidence that sense of body ownership can be induced on a bird avatar that is equal to a human avatar by synchronous condition. Flying condition was significantly higher than the synchronous condition. However, there was no interaction effect between the avatar and movement factors. Therefore, flying action is not a unique condition for inducing a sense of body ownership to the bird.

However, we observed that the flying condition was higher than the synchronous condition in rating the body change score on the bird avatar. Furthermore, we obtained a result that showed the flying condition was higher than synchronous condition in rating features score. It suggests that flying action is a drastic factor, but not a match condition. This is supported by 9 out of 10 participant's comments "I experienced that as if I metamorphosed into a bird, but not an animal suit".

\section{Conclusion}

We investigated the conditions for inducing a sense of body ownership to a bird avatar using IVR. We conducted some experiments to evaluate motion synchronization and a bird's characteristic features such as flying action, short body and sound of flapping in the rating of the sense of body ownership. As a result, motion synchronization was a sufficient condition for inducing sense of body ownership even in a bird avatar. Additionally, flying action was significantly higher than motion synchronization. Thus, flying action can enhance the sense of body ownership. It is not unique condition for inducing a sense of body ownership on a bird avatar, although it is an ability characteristic of a bird. However, flying action was significantly higher than motion synchronization in the rating of real body change. Another bird's feature such as short body and sound of flapping did not affect the sense of body ownership. Flying action is not a unique condition for enhancing a sense of body ownership to a bird avatar. However, it gives us the feeling that our real body transforms into a bird.

\section{References}

[1] Botvinick, M., \& Cohen, J. (1998). Rubber hands' feel'touch that eyes see. Nature, 391, 756

[2] Peck, T., Seinfeld, S., Aglioti, S., \& Slater, M. (2013). Virtual reality embodiment can reduce implicit racial bias. Conscious Cogn, 22(3), 779-787.

[3] Banakou, D., Groten, R., \& Slater, M. (2013) Illusory ownership of a virtual child body causes overestimation of object sizes and implicit attitude changes. Proceedings of the National Academy of Sciences of the United States of America 110: Vol 31 (pp. 12846-12851).

[4] Tsakiris, M. (2010). My body in the brain: A neurocognitive model of body-ownership. Neuropsychologia, 48, 703-712

[5] Sun, J. A., Joshua, B., Elise, O., Kristine, L. N., Kata, M., \& Jeremy B. (2016). Experiencing nature: Embodying animals in immersive virtual environments increases inclusion of nature in self and involvement with nature. Journal of Computer Mediated Communication, 1-21

[6] Gonzalez-Franco, M., Perez-Marcos, D., Spanlang, B., \& Slater, M. (2010). The contribution of real-time mirror reflections of motor actions on virtual body ownership in an immersive virtual environment. 
IEEE Virtual Reality (IEEE, Waltham, MA), 111-114.

[7] Lugrin, J. L., Latt, J., \& Latoschik, M. E. (2015). Anthropomorphism and illusion of virtual body ownership. Proceedings of International Conference on Artificial Reality and Telexistence Eurographics Symposium on Virtual Environments.

[8] Moseley, G. L., Olthof, N., Venema, A., Don, S., Wijers, M., \& Gallace, A., et al. (2008). Psychologically induced cooling of a specific body part caused by the illusory ownership of an artificial counterpart. Proceedings of the National Academy of Sciences (pp. 13169-13173)

[9] Armel, K. C., \& Ramachandran, V. S. (2003). Projecting sensations to external objects: Evidence from skin conductance response. Proceedings of the Royal Society London B Biological Sciences (pp. 14991506).

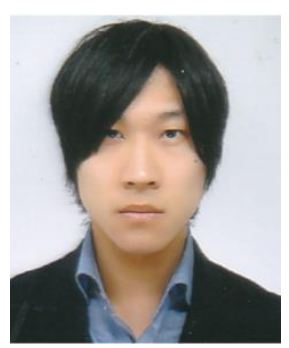

Akimi Oyanagi is a student at the Toyohashi University of Technology. He received the master of computer science in 2017. He is working on virtual reality psychology, and augmented human.

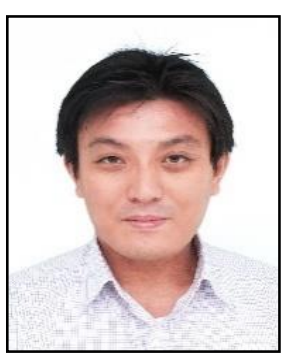

Ren Ohmura is a lecturer at Department of Computer Science and Engineering, Toyohashi University of Technology, Japan. He received the bachelor of electric engineering, master of computer science, and Ph.D. degrees from Keio University in 1999, 2001, and 2004, respectively. He is working on wearable computing, ubiquitous computing, IoT, context aware systems, and human-robot interaction. 